\title{
Measurements of continuous spectra of atmospheric absorption coefficients from UV to NIR via optical method
}

\author{
S. Mogo, ${ }^{1,2}$ V. E. Cachorro, ${ }^{2}$ M. Sorribas, ${ }^{2,3}$ A. de Frutos, ${ }^{2}$ and R. Fernández ${ }^{4}$ \\ Received 9 March 2005; revised 17 May 2005; accepted 7 June 2005; published 7 July 2005.
}

[1] In this study we extend from the visible to the UV a traditional method for studying the radiative properties of black carbon. We apply this measuring possibility to a practical study carried out in the Southern Iberian Peninsula during summer 2004. Using an optical technique based on the integrating sphere method, absorption coefficients, $\sigma_{a}$, were determined in the visible and in the ultraviolet regions of spectrum. The obtained values are representative of remote areas including during desert episodes that reach this area. To analyse the spectral shape of $\sigma_{a}$ we derived a parameter $\left(\alpha_{a}\right)$ which is the slope of the linear fit of the log$\log$ plot of $\sigma_{a}$ versus $\lambda$. This parameter is similar to the Angstrom exponent used for extinction. The reported $\alpha_{a}$ values for the UV appear as very original data in the literature. Citation: Mogo, S., V. E. Cachorro, M. Sorribas, A. de Frutos, and R. Fernández (2005), Measurements of continuous spectra of atmospheric absorption coefficients from UV to NIR via optical method, Geophys. Res. Lett., 32, L13811, doi:10.1029/2005GL022938.

\section{Introduction}

[2] Many of the difficulties in assessing an aerosols radiative net effect arises from a deficient evaluation of the absorption coefficient, usually much smaller than the scattering coefficient. The decomposition of the aerosol extinction into the two contributions, absorption and scattering, is not only difficult but also very imprecise, especially in what concerns the absorption coefficient. Measurements of the absorption coefficient are relatively scarce [Bond et al., 1999], at least, when compared with the abundance of scattering data. Besides, most of the existing data is obtained through filter based methods which derive absorption from the change in light transmission through a filter where the particles are collected. These methods include the integrating plate [Lin et al., 1973; Horvath, 1997], the integrating sphere [Fischer, 1973; Hitzenberger et al., 1996], the PSAP (Particle Soot Absorption Photometer) or the aethalometer [Hansen et al., 1982]. Other methods are the photoacoustic [Adams, 1988; Arnott et al., 1999] or the calorimetric [Hänel and Hillenbrand, 1989] methods.

[3] Black carbon is one of the most abundant lightabsorbing components of aerosols [Hitzenberger et al.,

\footnotetext{
${ }^{1}$ Departamento de Física, Universidad da Beira Interior, Covilhã Portugal. Spain.

${ }^{2}$ Grupo de Óptica Atmosférica, Universidad de Valladolid, Valladolid,

${ }^{3}$ Instituto Nacional de Técnica Aeroespacial, Mazagón, Spain.

${ }^{4}$ Universidad de Huelva, Huelva, Spain.
}

Copyright 2005 by the American Geophysical Union. 0094-8276/05/2005GL022938\$05.00
1996]. Its absorption coefficient is substantially higher than other species at the wavelengths we are working with: ultraviolet and visible. On the other hand, black carbon can be mixed with other species in different states of aggregation, internal mixture, external mixture, or core-shell structure, with significant changes in the optical properties [Chandra et al., 2004]. For example, the absorption coefficient of an internal mixture of elemental carbon with nonabsorbent particles is larger than the absorption coefficient of an external mixture [Horvath, 1993]. For a more complete understanding of black carbon behavior in the atmosphere it is necessary to gather a large and continuous time set of observations, especially in the ultraviolet region of spectrum, since very few values are found in the literature, except those measured with aethalometers [Alfaro et al., 2004; Fialho et al., 2005]. A reliable determination of the absorption coefficient of aerosols (hence the complex refractive index or the single scattering albedo) in the UV region is now one of the major challenges in aerosol studies. Here, we show the absorption coefficient determination and also the values associated to the spectral shape by means of a parameter similar to the Angstrom exponent, $\alpha$, used for extinction.

[4] The present work was carried out within the field of a larger intensive aerosol characterization campaign organized in summer 2004 at El Arenosillo. The main goal of this campaign was a comprehensive physical and chemical characterization of local aerosol. Several instruments for aerosol characterization were put together simultaneously: a scanning mobility particle sizer (SMPS) and an aerodynamic particle sizer (APS) for numerical size particle distribution in fine and coarse fractions respectively; two high volume sampler systems for geochemical analysis by various methods and a CIMEL photometer for columnar optical aerosol properties. Finally a cascade impactor with four stages was used in this work and its filters were analyzed for absorption coefficient determination.

\section{Description of the Experiment}

\subsection{Sampling Location}

[5] The aerosol samples were collected in summer 2004, from July 15 th to October 15 th, at the Atmospheric Sounding Station "ESat - El Arenosillo", $\left(37.10^{\circ} \mathrm{N}, 6.70^{\circ} \mathrm{W}\right)$, National Institute of Aerosol Technology (INTA), $\approx 30 \mathrm{~km}$ from Huelva, Spain.

[6] This is a non-urban station located in the neighborhood of a nature-protected area, the national park of Doñana; the proximity of Huelva town with a strong industrial belt represents the main source of pollution in the collected samples. Huelva is a medium size town with $\approx 160000$ inhabitants, located in the south-west of the 
Table 1. Characteristics of DEKATI PM10 Impactor ${ }^{\mathrm{a}}$

\begin{tabular}{ccc}
\hline Stage & Size range $/ \mu \mathrm{m}$ & Modal regime \\
\hline 0 & $<1$ & fine \\
1 & $1-2.5$ & coarse \\
2 & $2.5-10$ & coarse \\
3 & $>10$ & coarse \\
\hline
\end{tabular}

${ }^{\mathrm{a}}$ Air flow: $16.5 \mathrm{l} / \mathrm{min}$. Sizes are aerodynamic equivalent size.

Iberian Peninsula and with maritime climate. The emissions from its large industrialized area are mixed with urban emissions and also with frequent arrivals of African desert dust. Emission from the nearest main road, at $\approx 500 \mathrm{~m}$ from the sample collecting site, are also present. Predominant wind direction in summer, during day time, is south-west (from the sea), 23\%. During the night predominance is from north-west (from inland), 13\%. There is approximately $30 \%$ of calm days during this season.

\subsection{Experimental Procedure}

[7] The aerosol was collected over Millipore polycarbonate membrane filters with $0.2 \mu \mathrm{m}$ pores using a Dekati PM10 impactor. The impactor had a constant flow rate of $16.5 \mathrm{l} / \mathrm{min}$ and the sampling time was between 24 and $72 \mathrm{~h}$. The impactor has four stages and its aerodynamic cut-off diameters are presented in Table 1.

[8] The filters were weighed before and after collection and care was taken in order to eliminate electrostatic charges from the filters before weighing.

[9] For determination of the absorption coefficients we developed and built an integrating sphere photometer. The working principle was first used for this purpose by [Fischer, 1973]. We enhanced the illumination and detection systems of the original method.

[10] The exposed filters were dissolved in chloroform and the solution was put into a quartz cell located in the center of the integrating sphere, Figure 1. The dissolution of the polycarbonate filter leaves the aerosol particles in suspension. A stable suspension of the aerosols was obtained using ultrasound. To minimize errors due to the differences between filters the light transmission coefficient through the blank filter was evaluated as the average of 20 blank filters.

[11] In spite of being a destructive technique it has the advantage of approaching the aerosol measurement conditions to the natural conditions of the atmospheric aerosols, when compared to the option of using the particles trapped in the filters. Furthermore, the real part of the refractive index of chloroform is nearly the same as that of suspended particles, so the light scattering is highly reduced. The effect of changing the medium from chloroform to air is a reduction in the effective refractive index. As a consequence, light absorption is reduced by a maximum of $5 \%$ [Hitzenberger et al., 1996], therefore no refractive index correction is applied to the results reported in this paper.

[12] A sketch of the system used is shown in Figure 1. The light source is a $450 \mathrm{~W}$ xenon lamp. Between the light and the integrated sphere there is a double monochromator for illuminating the quartz cell with monochromatic light. In this way the wavelength can be chosen according to the diffraction gratings of the monochromator. The detection system is a simple photosensor. The monochromator spec- tral range is $320-800 \mathrm{~nm}$ with $1 \mathrm{~nm}$ resolution, but for a quick measurement we took a $10 \mathrm{~nm}$ sampling step. With this approach continuous spectra in the ultraviolet, visible and near infrared spectral regions were obtained.

[13] Aerosol absorption coefficients $\left(\sigma_{a}\right)$ of particle samples were evaluated by measuring the apparent transmission $(T(\lambda))$ of the light source beams through the quartz cell:

$$
T(\lambda)=I_{f}(\lambda)\left(I_{0}(\lambda)\right)^{-1}
$$

where $I_{f}(\lambda)$ and $I_{0}(\lambda)$ are the intensities of light passing through the quartz cell with a sampling filter dissolved and with a blank filter dissolved, respectively, for each wavelength. Assuming that the attenuation of the beam follows the Beer-Lambert law, one has:

$$
I_{f}(\lambda)=I_{0}(\lambda) e^{-x \sigma_{a}(\lambda)}
$$

where $x$ is the optical path length, which can be determined from the volume of the sampled air, $V$, and the effective cross-section area of the cell, $A$. The absorption coefficient can be determined with the $I_{0}(\lambda), I_{f}(\lambda), V$ and $A$ values:

$$
\sigma_{a}(\lambda)=-\frac{A}{V} \ln \frac{I_{f}(\lambda)}{I_{0}(\lambda)}
$$

[14] The mass absorption specific coefficient of fine particles, $B_{a}(\lambda)$, can be obtained by $B_{a}(\lambda)=\sigma_{a}(\lambda)$. $\left(M_{\mathrm{PM} 1}\right)^{-1}$, where $M_{\mathrm{PM} 1}$ is the mass concentration of fine particles (aerodynamic size bellow $1 \mu \mathrm{m}$ ).

\section{Results and Discussion}

[15] The evolution during the campaign of time series of the mass concentrations of PM10 and PM1 are shown in Figure $2 \mathrm{a}$. The ratio of coarse to fine particles, $\mathrm{C} / \mathrm{F}$, is also shown. The PM10 concentration ranged between $7.4 \mu \mathrm{g}$. $\mathrm{m}^{-3}$ and $119.7 \mu \mathrm{g} \cdot \mathrm{m}^{-3}$, the average value was $28.3 \mu \mathrm{g}$. $\mathrm{m}^{-3}$. The PM1 concentration ranged between $3.8 \mu \mathrm{g} \cdot \mathrm{m}^{-3}$ and $43.6 \mu \mathrm{g} \cdot \mathrm{m}^{-3}$, the average value was $16.5 \mu \mathrm{g} \cdot \mathrm{m}^{-3}$. The smaller values registered are representative of the local rural background. The higher values belong to Saharan dust events that occurred during the campaign. The strongest of those events took place in Jul 22, Aug 22, Sep 09 and Oct 06 . The event of Jul 22 was exceptionally high, it was the most intense registered in Spain with presently used modern techniques of detection (Cimel and backtrajectory data).

[16] Size distribution of local aerosols is characterized by its bimodal feature, with the coarse fraction mostly composed by dust and mineral particles while the fine fraction is more associated with air pollution. Usually the ratio $\mathrm{C} / \mathrm{F}$ is

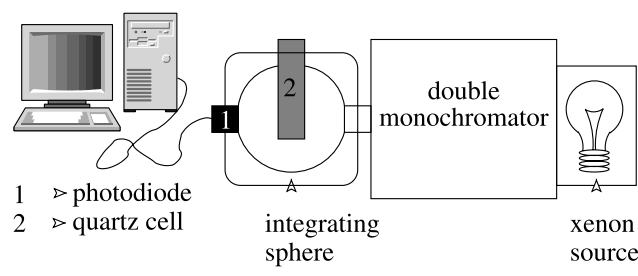

Figure 1. The experimental apparatus. 

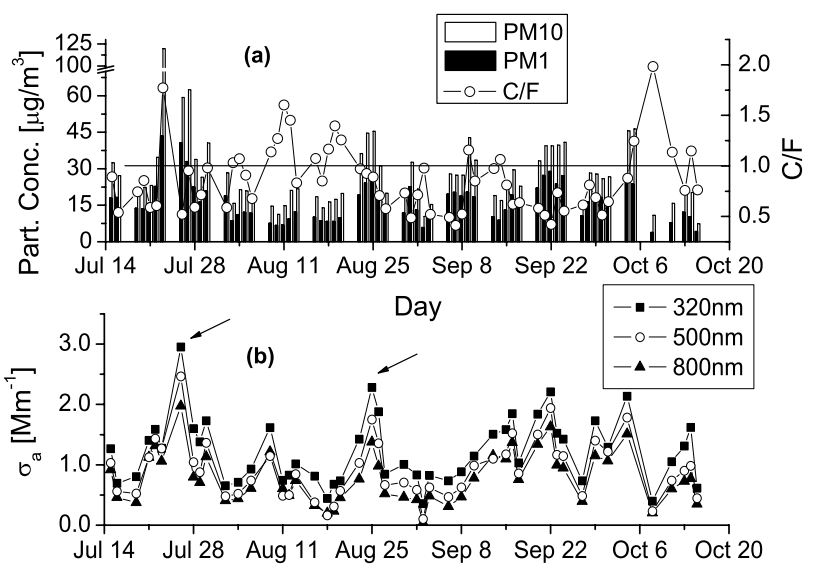

Day

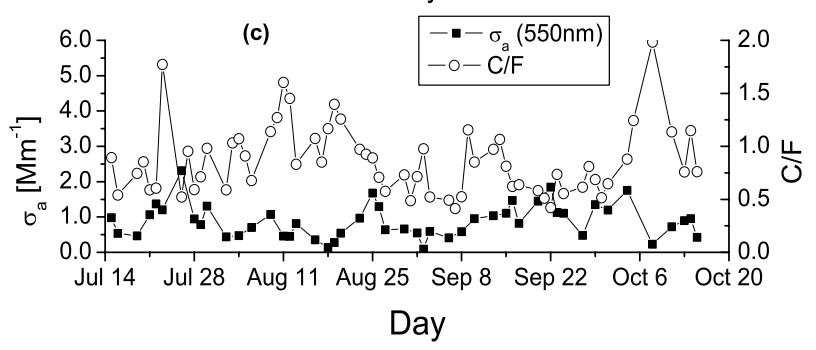

Figure 2. (a) Aerosols concentrations during the field campaign and $\mathrm{C} / \mathrm{F}$ ratio (64 samples). Note the break of $\mathrm{Y}$ axis. (b) Time series of the $\sigma_{a}$ for $320 \mathrm{~nm}(-\mathbf{-}), 500 \mathrm{~nm}$ $\left(-0^{-}\right)$and $800 \mathrm{~nm}\left(-\boldsymbol{\Delta}_{-}\right)$. Saharan dust events identified by arrows. (c) Variation of $\sigma_{a}$ for $550 \mathrm{~nm}(--)$ and ratio $\mathrm{C} / \mathrm{F}(-\mathrm{O}-)$.

smaller than 1.0, due to predominance of fine particles. Nevertheless, the ratio $\mathrm{C} / \mathrm{F}$ increases with the arrival of a dust event, showing predominance of the coarse fraction in this situation.

[17] For comparative studies we found in the literature many values of the mass absorption specific coefficient instead of the absorption coefficient. In our opinion, comparing the absorption coefficient has two important advantages. First, it does not depend on the mass concentration which is a very variable element, thus presenting major difficulty for long term comparison. Second, the errors associated with the mass absorption specific coefficient

Table 2. Mean, Standard Deviation, Minimum and Maximum Daily Levels of $\sigma_{a}$ for Selected Wavelengths According to Those Used by Other Instrumentation (Cimel, TOMS) in Order to Facilitate Comparison of Data

\begin{tabular}{ccccc}
\hline & Mean & $(\mathrm{Std})$ & Minimum & Maximum \\
\cline { 2 - 5 } Wavelength [nm] & & \multicolumn{3}{c}{$\sigma_{a}\left[\mathrm{Mm}^{-1}\right]$} \\
\hline 320 & 1.22 & $(0.55)$ & 0.36 & 2.95 \\
340 & 1.16 & $(0.55)$ & 0.29 & 2.92 \\
360 & 1.13 & $(0.54)$ & 0.26 & 2.83 \\
380 & 1.07 & $(0.53)$ & 0.23 & 2.74 \\
440 & 1.00 & $(0.52)$ & 0.14 & 2.60 \\
500 & 0.93 & $(0.49)$ & 0.10 & 2.46 \\
550 & 0.88 & $(0.47)$ & 0.09 & 2.31 \\
670 & 0.77 & $(0.43)$ & 0.03 & 2.10 \\
750 & 0.76 & $(0.42)$ & 0.05 & 1.99 \\
800 & 0.78 & $(0.42)$ & 0.04 & 1.97 \\
\hline
\end{tabular}

determination is considerably higher than those of the absorption coefficient determination because of the added mass concentration error. Therefore, here we considered more interesting to analyse the variability of the absorption coefficient.

[18] For the visible wavelength of $550 \mathrm{~nm}$ [Heintzenberg and Bussemer, 2000] presented values between 5-80 $\mathrm{Mm}^{-1}$ for aerosols in Melpitz, Germany. Arnott et al. [2003] reported a maximum value of $2.1 \mathrm{Mm}^{-1}$ for Big Bend National Park in South Texas, USA. El Arenosillo values are typical of remote areas and are reported in Figure $2 b$ which shows time series of the absorption coefficients, during the measurement period, for three representative wavelengths, $320 \mathrm{~nm}$ in the UV range, $500 \mathrm{~nm}$ in the visible and $800 \mathrm{~nm}$ in the infrared. As we have continuous spectra from 320 to $800 \mathrm{~nm}$, we show in Table 2 the mean, minimum and maximum (standard deviation in parentheses) for ten wavelengths covering the above referred spectral range. For our limit wavelengths, $320 \mathrm{~nm}$ and $800 \mathrm{~nm}$, the average absorption coefficients measured are $1.22 \mathrm{Mm}^{-1}$ and $0.78 \mathrm{Mm}^{-1}$. For a wavelength in the middle of the visible spectrum, $550 \mathrm{~nm}$ the average absorption coefficient is $0.93 \mathrm{Mm}^{-1}$. However other values can be considered as relevant as those of UV wavelengths as can be seen in the table.

[19] The variation of absorption coefficient for $550 \mathrm{~nm}$ and the ratio $\mathrm{C} / \mathrm{F}$ are presented in Figure $2 \mathrm{c}$ for simultaneous analysis. By comparing $\sigma_{a}$ with $\mathrm{C} / \mathrm{F}$ we intend to find a relationship between the absorption coefficients determined and the kind/size of the particles involved. Through careful observation of curve shapes we may conclude that, in general, each increase of the absorption coefficient coincides with a decrease of the ratio $\mathrm{C} / \mathrm{F}$. Exceptions to this situation happen when there is a Saharan dust event. Let us choose the strong dust event of Jul 22 for a more complete analysis. We observed an increase of absorption coefficients right after the beginning of the dust event. The ratio $\mathrm{C} / \mathrm{F}$ reaches the highest value on July 22 , while absorption coefficient reaches its highest value on Jul 26. The peak of $\sigma_{a}(---)$ is shifted to the right with respect to the peak $\mathrm{C} / \mathrm{F}(-\mathrm{O}-)$, Figure 2c. This $\sigma_{a}$ increase following the $\mathrm{C} / \mathrm{F}$ increase could probably be explained by the coagulation of fine black carbon particles with dust.

[20] In Figure 3 we present the spectral shape variation of absorption coefficients throughout the spectrum. The three

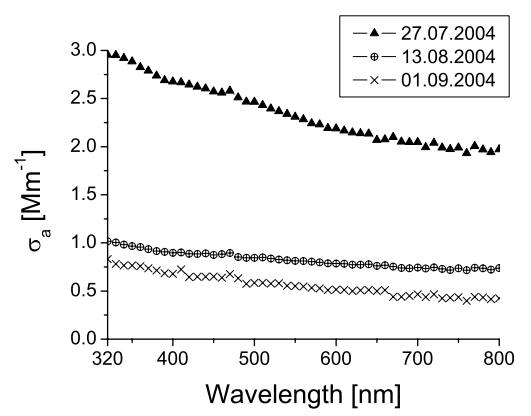

Figure 3. $\sigma_{a}$ variation along the spectrum for three representative days: a particularly high, Jul 27 (А); a day of low value, Sep $01(\times)$; and a day of medium level, Aug $13(\oplus)$. 


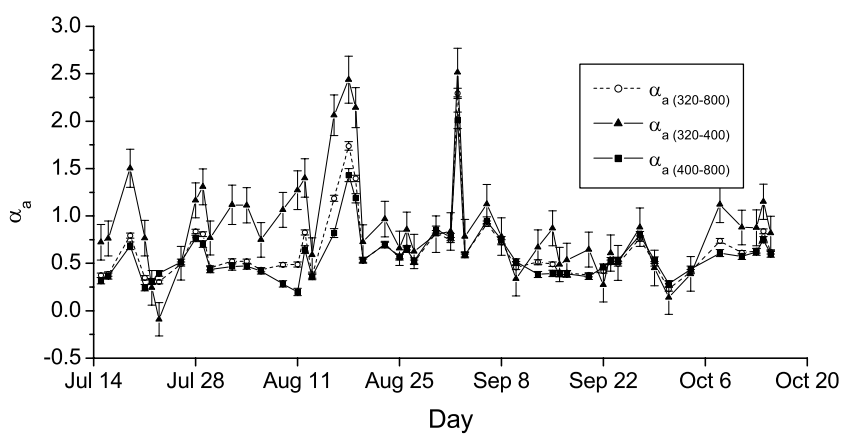

Figure 4. Alpha parameter calculated as the slope of the linear fit of the log-log plot of the absorption coefficient versus the wavelength. $\alpha_{320-400}(-\mathbf{\Delta}-)$ was obtained for the $\mathrm{UV} ; \alpha_{400-800}\left(-\mathbf{-}_{-}\right)$for the visible; and $\alpha_{320-800}(-\circ-)$ for all the $320-800 \mathrm{~nm}$ interval.

curves presented belong to three example days: one exceptionally high (Jul 27), one particularly low (Sep 01) and the last with the normal atmospheric condition of the area (Aug 13). As shown in Figure 3 the absorption coefficient decreases with wavelength, the smaller the wavelength the higher the absorption coefficient. This decrease, however, is not very pronounced, reaching a maximum difference between $320 \mathrm{~nm}$ and $800 \mathrm{~nm}$ of $0.98 \mathrm{Mm}^{-1}$ and a minimum difference of $0.14 \mathrm{Mm}^{-1}$, during our measurement period. The average difference between absorption coefficients at $320 \mathrm{~nm}$ and $800 \mathrm{~nm}$ was $0.44 \mathrm{Mm}^{-1}$. Our daily detection limit is $0.003 \mathrm{Mm}^{-1}$ and a rough estimate of the measurement error does not exceed $10 \%$. This estimate is higher in the UV and NIR because of the lower source intensities in those regions, resulting in more noisy signal levels.

[21] For a more detailed analysis of the variations of the spectral shape we can define a parameter equivalent to the Angstrom exponent, $\alpha_{a}$, similar to that used for extinction [Bond, 2001]: $\sigma_{a}=K \lambda^{-\alpha_{a}}$, where $K$ is a constant, $\lambda$ is the wavelength of light, and the subscript $a$ indicates the absorption by particles. To determine the alpha coefficient we made a fit of the $\log$-log plot of $\sigma_{a}$ versus the wavelength. We considered three different alpha coefficients, one obtained taking the spectral range from 320 to $800 \mathrm{~nm}$, other representative of the visible spectral range (400$800 \mathrm{~nm})$ and another one for the UV range (320-400 nm). In Figure 4 we illustrate the evolution of these three alpha coefficients.

[22] [Bond et al., 1999] reported values of $\alpha_{a}$ between 1.7 and 2.5 for industrial combustion of lignite, and absorption efficiency measured at $450 \mathrm{~nm}, 550 \mathrm{~nm}, 750 \mathrm{~nm}$ and $1000 \mathrm{~nm}$. [Rosen et al., 1979] measured $\alpha_{a}=1.0$ in an urban environment, for $633 \mathrm{~nm}$. In our work, alpha values obtained for the $320-800 \mathrm{~nm}$ region are in the range $0.2-$ 2.5 with an average value of 0.6 (standard deviation 0.4 ). In the UV values are generally higher but with more extreme values $(-0.1$ to 2.5$)$. One negative value was obtained, which could be explained because of the high sensibility of this parameter to the absorption spectral variation. We must be aware of the high uncertainty in the determination of this parameter which could explain the extreme values. In our work, alpha values are representative of different situations that occurred during the 3 months of campaign; the 0.6 average value seems to be characteristic of a clean atmosphere with black carbon as trace component.

\section{Concluding Remarks}

[23] The study case we chose to apply our technique to determine $\sigma_{a}$ is very interesting due to its peculiar location in a nature protected area that sometimes receives samples from a nearby heavily industrialized area and the arrival of frequent desert aerosol outbreaks, allowing a huge range of aerosol sample types. The reported values of $\sigma_{a}$ are relatively low, presenting in average $1.22 \mathrm{Mm}^{-1}, 0.93 \mathrm{Mm}^{-1}$ and $0.78 \mathrm{Mm}^{-1}$ for UV $(320 \mathrm{~nm})$, visible $(500 \mathrm{~nm})$ and NIR $(800 \mathrm{~nm})$ respectively. The values in the UV are in average $24-25 \%$ higher than in the visible region of spectrum. Measuring $\sigma_{a}$ with high spectral resolution allowed us to determine reliable $\alpha_{a}$ values. These values are very innovative in the literature giving news about aerosol absorption characterization. As we observed a large range of $\alpha_{a}$ values, it would be interesting to make a more extensive comparison of these values in a near future. All the reported values are sufficiently representative of non-urban sites in Spain where the arrival of desert dust aerosol is relatively frequent.

[24] Acknowledgment. This study was supported by CYCIT Ref. REN2002-00966/CLI.

\section{References}

Adams, K. (1988), Real time in situ measurements of optical absorption in the visible via photoacoustic spectroscopy: Evaluation of photoacoustic cells, Appl. Opt., 27(19), 4052-4056.

Alfaro, S., S. Lafon, J. Rajot, P. Formenti, A. Gaudichet, and M. Maillé (2004), Iron oxides and light absorption by pure desert dust: An experimental study, J. Geophys. Res., 109, D08208, doi:10.1029/ 2003JD004374.

Arnott, W., H. Moosmüller, C. Rogers, T. Jin, and R. Bruch (1999), Photoacoustic spectrometer for measuring light absorption by aerosol: Instrument description, Atmos. Environ., 33, 2845-2852.

Arnott, W., H. Moosmüller, P. Sheridan, J. Ogren, R. Raspet, W. Slaton, J. Hand, S. Kreidenweis, and J. L. Collett Jr. (2003), Photoacoustic and filter-based ambient aerosol light absorption measurements: Instrument comparisons and the role of relative humidity, J. Geophys. Res., 108(D1), 4034, doi:10.1029/2002JD002165.

Bond, T. (2001), Spectral dependence of visible light absorption by carbonaceous particles emitted from coal combustion, Geophys. Res. Lett., 28(21), 4075-4078.

Bond, T., T. Anderson, and D. Campbell (1999), Calibration and intercomparison of filter-based measurements of visible light absorption by aerosols, Aerosol Sci. Technol., 30, 582-600.

Chandra, S., S. Satheest, and J. Srinivasan (2004), Can the state of mixing of black carbon aerosols explain the mistery of 'excess' atmospheric absorption?, Geophys. Res. Lett., 31, L19109, doi:10.1029/ 2004GL020662.

Fialho, P., A. Hansen, and R. Honrath (2005), Absorption coefficients by aerosols in remote areas: A new approach to decouple dust and black carbon absorption coefficients using seven-wavelength aethalometer data, J. Aerosol Sci., 36, 267-282.

Fischer, K. (1973), Mass absorption coefficients of natural aerosol particles in the 0,4 to $2,4 \mu \mathrm{m}$ wavelength interval, Contrib. Atmos. Phys., 46, 89100 .

Hänel, G., and C. Hillenbrand (1989), Calorimetric measurement of optical absorption, Appl. Opt., 28(3), 510-516.

Hansen, A., H. Rosen, and T. Novakov (1982), Real-time measurement of the absorption coefficient of aerosol particles, Appl. Opt., 21(17), 30603062 .

Heintzenberg, J., and M. Bussemer (2000), Development and application of a spectral light absorption photometer for aerosol and hydrosol samples, J. Aerosol Sci., 31(7), 801-812.

Hitzenberger, R., U. Dusek, and A. Berner (1996), Black carbon measurements using an integrating sphere, J. Geophys. Res., 101, 19,60119,606 . 
Horvath, H. (1993), Atmospheric light absorption-A review, Atmos. Environ., 27A, 293-317.

Horvath, H. (1997), Experimental calibration for aerosol light absorption measurements using the integrating plate method-Summary of the data J. Aerosol Sci., 28(7), 1149-1161.

Lin, C., M. Baker, and R. Charlson (1973), Absorption coefficient of atmospheric aerosol: A method of measurement, Appl. Opt., 12(6), 13561363.

Rosen, H., D. Hansen, L. Gundel, and T. Novakov (1979), Identification of the graphitic carbon component of source and ambient particulates by Raman spectroscopy and an optical attenuation technique, in Carbona- ceous Particles in the Atmosphere, pp. 229-232, Lawrence Berkeley Natl. Lab., Berkeley, Calif.

V. E. Cachorro, A. de Frutos, and M. Sorribas, Grupo de Óptica Atmosférica, Universidad de Valladolid, E-47071 Valladolid, Spain.

R. Fernández, Universidad de Huelva, E-21071 Huelva, Spain.

S. Mogo, Departamento Física Teórica, Atómica y Óptica, Facultad de Ciencias, Universidad de Valladolid, Prado Magdalena S/N, E-47071 Valladolid, Spain. (smogo@ubi.pt) 